\title{
Indexing Multimedia Databases
}

\author{
Christos Faloutsos
}

Copyright (C1994, by Christos Faloutsos. All Rights Reserved. 


\section{Contents}

1 PROBLEM - APPLICATIONS

3

2 FRAMEWORK

5

3 SPATIAL ACCESS METHODS 8 $3.1 \quad$ Space filling curves . . . . . . . . 11 3.2 Grid File . . . . . . . . . . . 14 $3.3 \quad$ R-trees . . . . . . . . . 17 3.4 Conclusions . . . . . . . . . . . . . 19

4 TIME SERIES 20

5 COLOR IMAGES $\quad \mathbf{2 7}$ 5.1 Color - features and distance function 28 $5.2 \quad$ Shapes .............. . . 32 5.3 Performance........... . . 33

6 3-D MEDICAL IMAGES 35

7 SUB-PATTERN MATCHING 39

8 CONCLUSIONS $\quad 47$ 


\section{PROBLEM - APPLICATIONS}

Multimedia System: A system that can store and retrieve objects/documents with text, voice, images, animation, slides show etc.

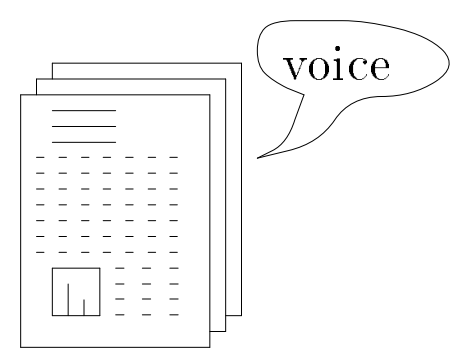

Problem definition:

- given a set of multimedia objects,

- find the ones containing a desirable pattern (or something similar to it)

GOALS: search by content

- Efficient and

- 'complete' (no false dismissals) 


\section{Applications:}

- time series: financial, marketing, ECGs, voice/sound

- images: education, art, medicine

- higher-d signals: scientific db (eg., meteorology, astrophysics), medicine, entertainment (video)

Sample queries:

- find companies whose stock prices move similarly

- find past days in which the solar magnetic wind showed patterns similar to today's pattern [Vas93]

- find X-rays similar to Smith's

- in LANDSAT images, find areas with cornfield colors

- find the average MRI brain scan of epileptics Similarity search, hypothesis testing, rule discovery, data mining [Agrawal et al., SIGMOD 93] [AIS93] 


\section{FRAMEWORK}

- Whole matching vs. Sub-pattern matching

- Range queries vs Nearest Neighbor

- All-pairs queries ('spatial joins')

Database

S1

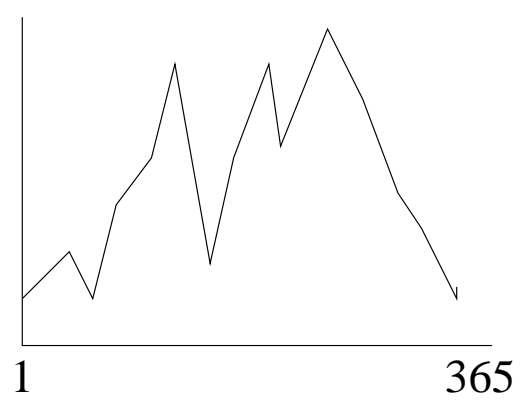

:

$\mathrm{Sn}$

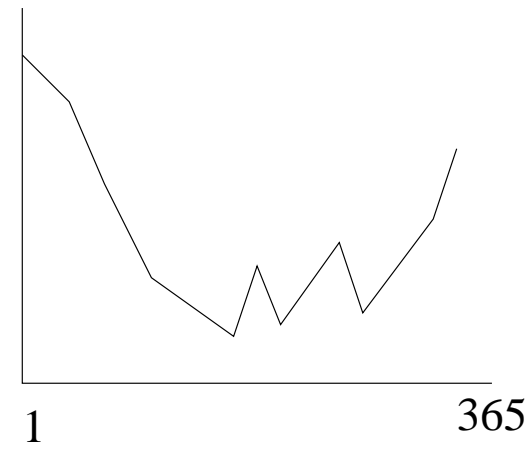

whole match query

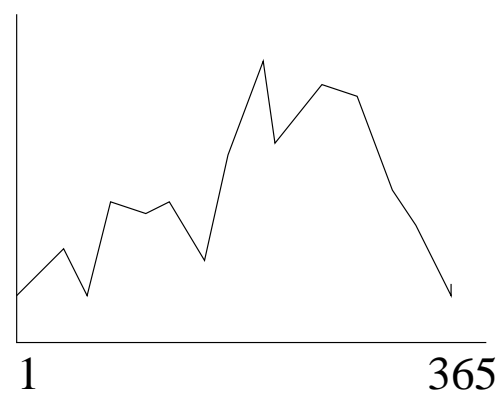

sub-sequence match

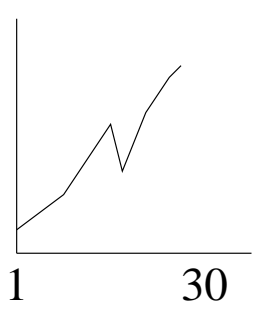

Distance function: by domain expert.

Eg., Euclidean

$$
\mathcal{D}(S, Q) \equiv\left(\sum_{i=1}^{l}|S[i]-Q[i]|^{2}\right)^{1 / 2}
$$

(similarly for vector fields) 
Database

S1

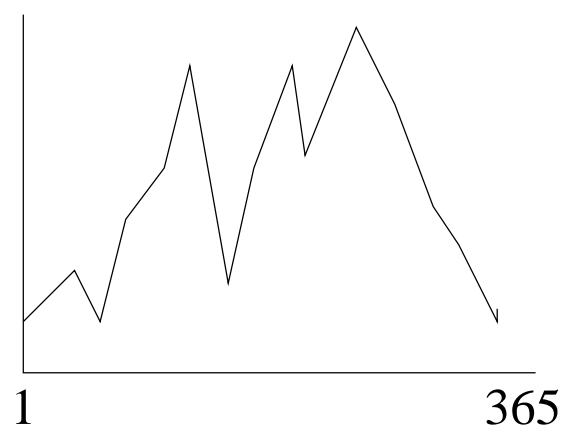

$\mathrm{Sn}$

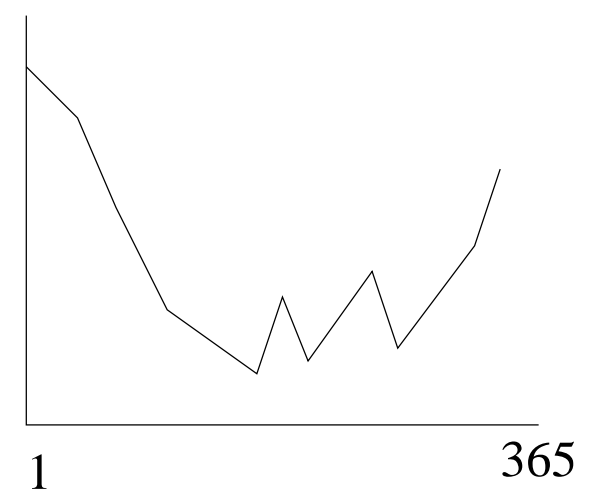

whole match query

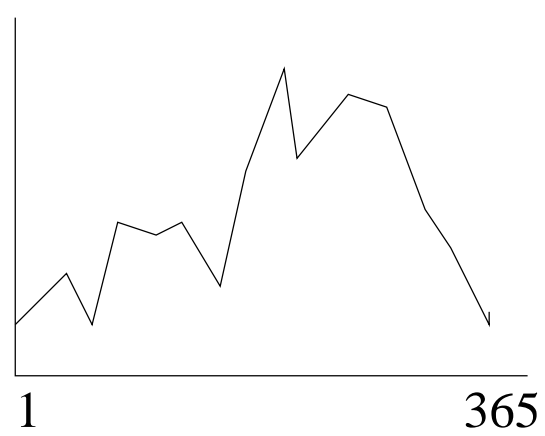

sub-sequence match

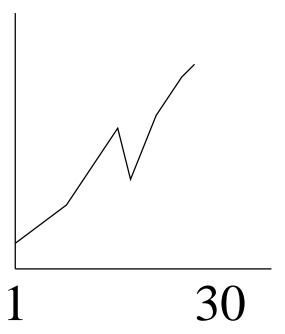




\section{Target method:}

- fast

- no false dismissals (false alarms are OK)

- dynamic (insertions/deletions/appends) 


\section{SPATIAL ACCESS METHODS}

Objects may be points, rectangles or arbitrary shapes. Queries:

- point queries,

- range queries,

- 'all-pairs'/spatial join queries [BKS93],

- nearest neighbor(s) queries [RKV95].

Applications

- Traditional data base systems.

- multimedia objects, after feature extraction [Jag91b]

- Cartography [Sam90a]

- Computer-Aided Design (CAD).

- Computer vision and robotics [BB82]

- Rule indexing in expert database systems [SSH86] 


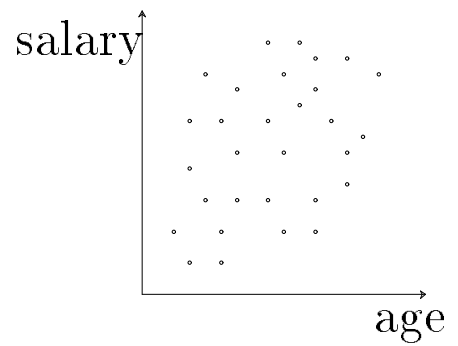

traditional data base

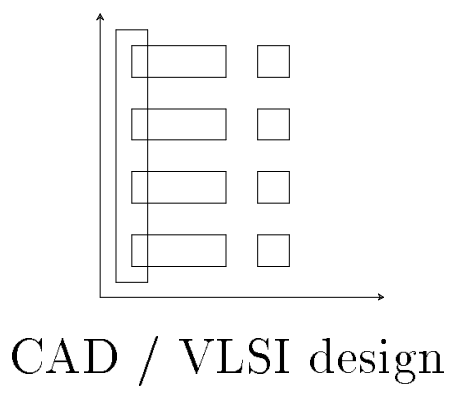

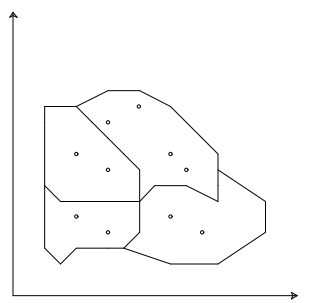

Cartographic data

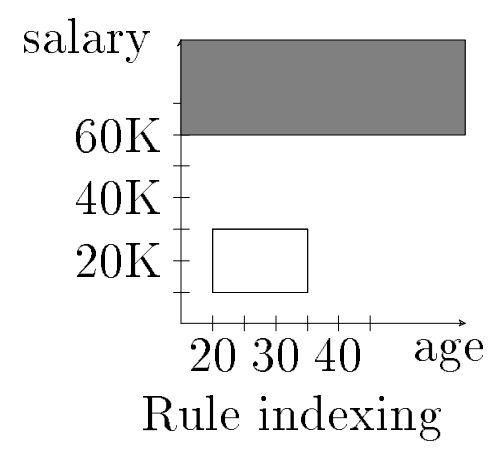

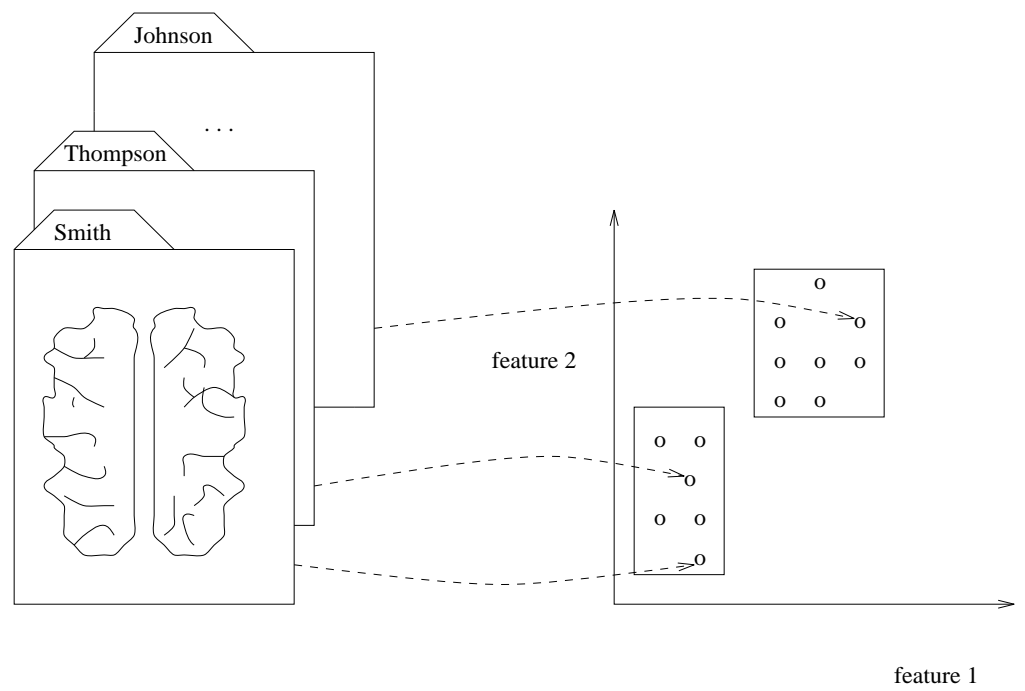




\section{Overview}

- space filling curves/z-ordering / linear quadtrees

- grid files

- R-trees 


\subsection{Space filling curves}

Proposed, among others, by. [Ore86] [SSN87] [OM88] [Ore89] [Ore90] [Sam90b]. Very similar linear quadtrees [Gar82].
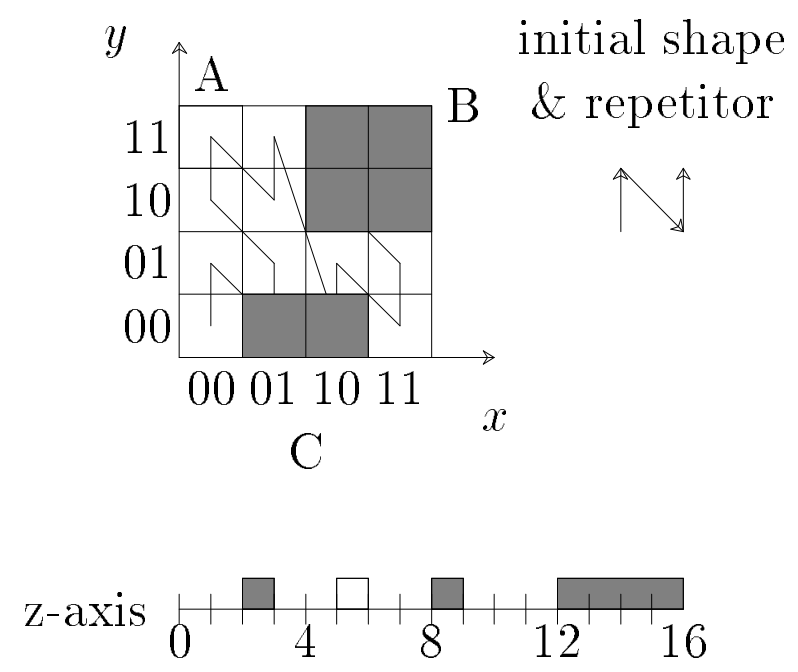

- $z_{A}=$ Shuffle ( "1,2,1,2", $\left.x_{A}, y_{A}\right)=$ Shuffle ("1,2,1,2", $00,11)=0101=(5)_{1} 0$

- $z_{B}=11$ (common prefix of all its blocks)

- $z_{C_{1}}=0010=2$

- $z_{C_{2}}=1000=8$ 


\section{NOTICE:}

- Relations using $z$ values $\Rightarrow$

- excellent integration of geometric data bases with relational ones

- fast processing of geometric queries, using index on the $z$ values

- used by the U.S. Bureau of Census - TIGER project [Whi81]

- BUT: regions give too many pieces, unless we use approximations (='redundancy' [Ore90]). 
Variations - improvements

- Best distance preserving mapping?

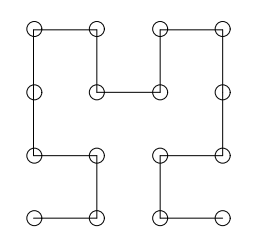

- Study of clustering properties of space filling curves:

- Exhaustive enumeration, [FR89b]

- formulas for partial match queries and $2 \times 2$ squares, [Jag90]

- closed formula for z-ordering [RF91] 


\subsection{Grid File}

Dynamic version of multi-attribute hashing [NHS84]

- 2 disk accesses for exact match queries

- symmetric with respect to the attributes

- adapting to non-uniform distributions

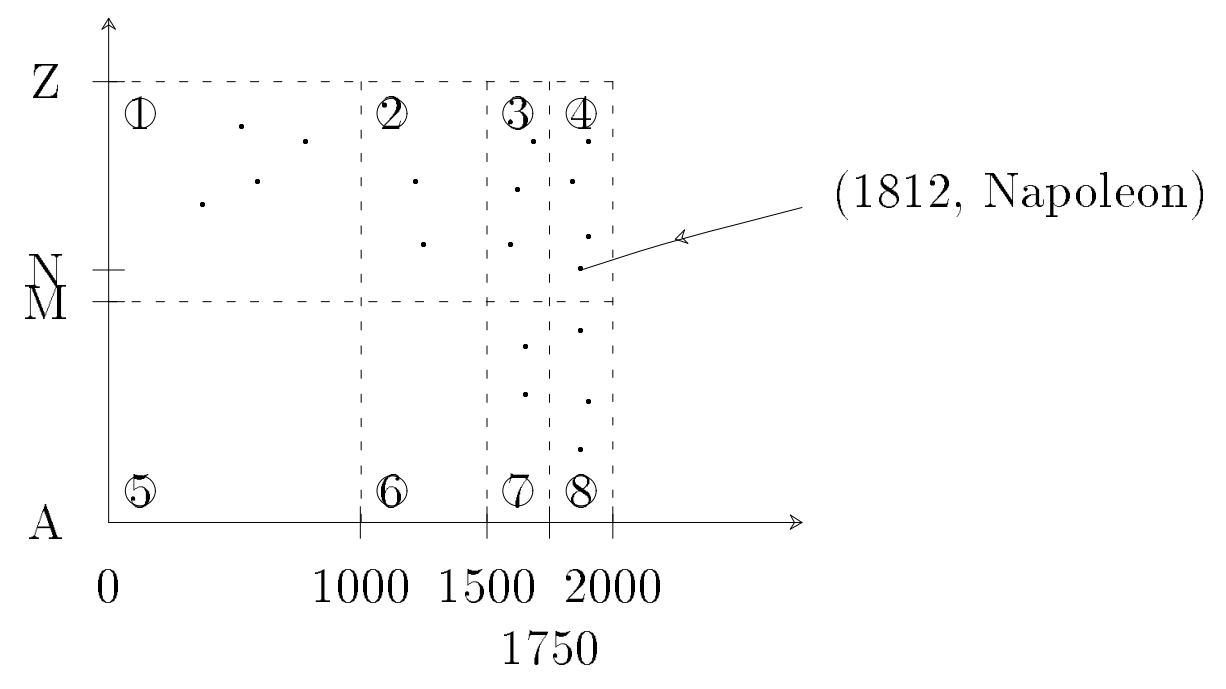

- Every cell $\rightarrow$ one disk page

- Cuts occur on predefined points $(1 / 2,1 / 4$ etc of each axis)

- Cuts cut all the way (as opposed to k-d-B-trees) 


\section{Implementation}

$\mathrm{y}$ - cutpoints

\begin{tabular}{|c|}
\hline $\mathrm{Z}$ \\
\hline $\mathrm{M}$ \\
\hline $\mathrm{A}$ \\
\hline
\end{tabular}

MAIN MEMORY

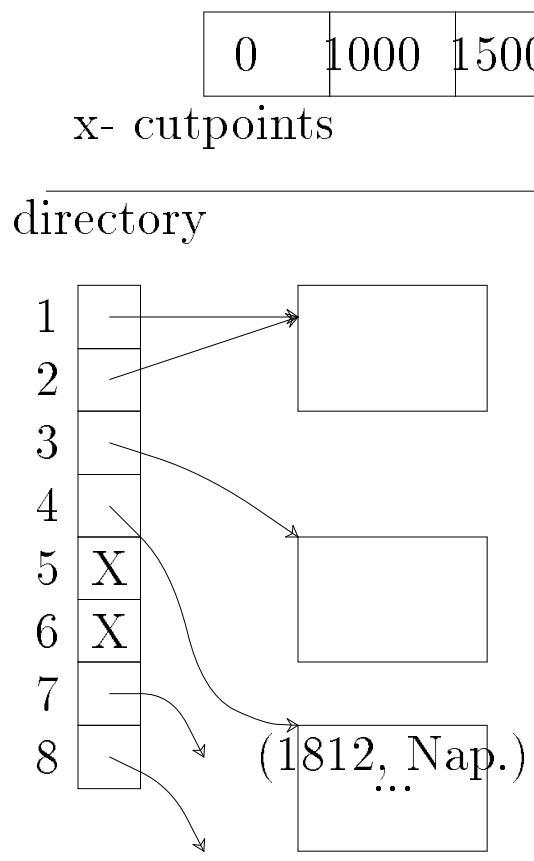

DISK 
BUT: correlated attributes

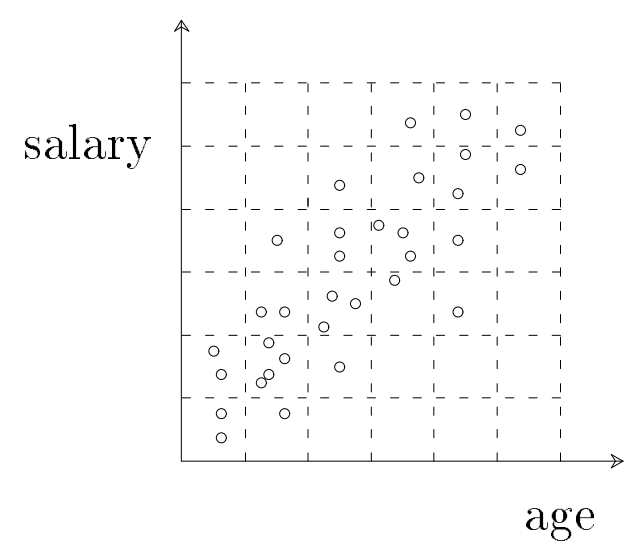

Solutions:

- Rotated grid file [HN83]

- tricell [FR89a]

Other Variations:

- Twin grid file [HSW88]

Notice that it handles points; rectangles can be handled by transforming them into points in 4-d [HN83] 


\subsection{R-trees}

Idea: Group points in parents - allow parents to overlap. [Gut84]

- balanced

$\bullet \geq 50 \%$ utilization
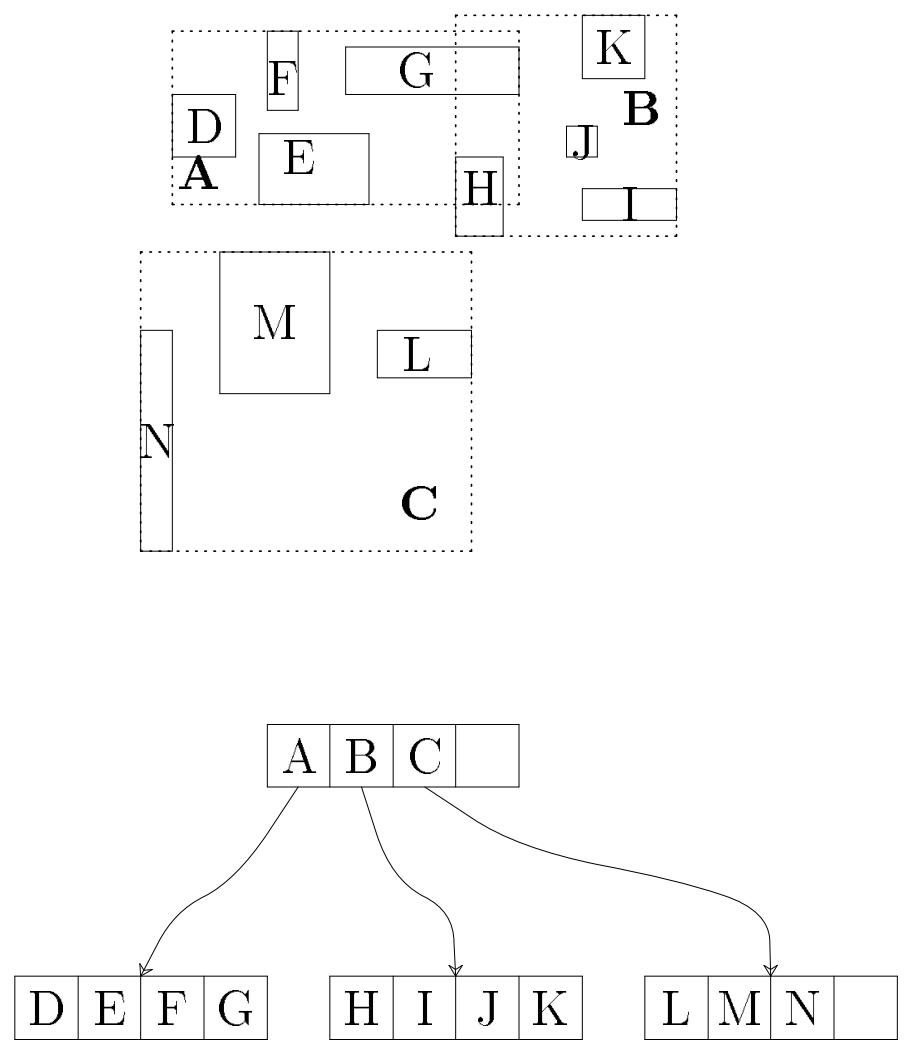

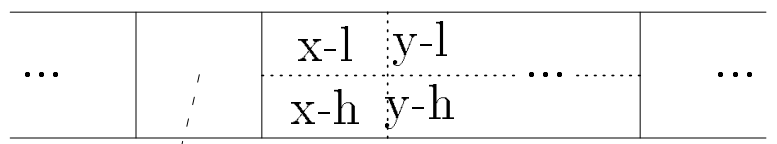

pointer

to child 
Variations / Improvements:

- Packed R-trees [RL85] [KF93] for static data.
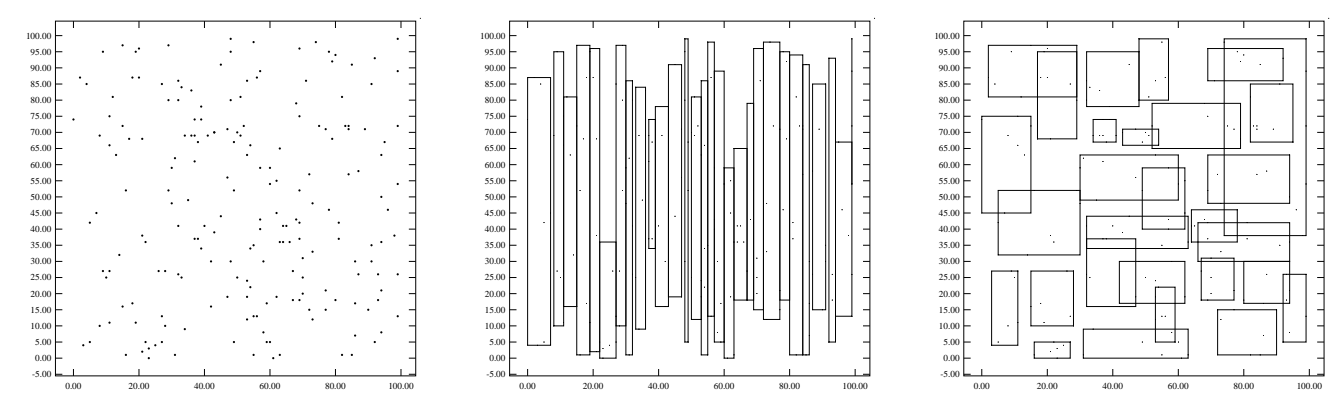

- cell trees [Gun86] introduce diagonal cuts, too.

- R+ trees: [FSR87] [SRF87] No overlap; balanced. Outperform R-trees when there are few large rectangles and several small ones.

- $R^{*}$-trees [BKSS90] Main idea: defer splitting, using forced-reinsert on $30 \%$ of the most remote rectangles.

- Hilbert R-trees [KF94] defer split, by pushing keys to the 'Hilbert' neighbor.

- Analysis for R-trees: Range queries [PSTW93]; using fractal dimensions [FK94] 


\subsection{Conclusions}

- Z-ordering (Linear quadtrees) and R-trees seem the most promising methods.

- R-trees are more robust for high-d spaces. 


\section{TIME SERIES}

[Agrawal et al., FODO 93] [AFS93]

Distance: Euclidean

Obvious solution: sequential scan.

Q: Something faster?

A: 'Quick-and-dirty' filter:

- extract $n$ features (numbers, eg., avg., etc.)

- map a sequence into a point in $n$-d feature space

- organize points with a Spatial Access Method (SAM) [Jag91b]

- discard false alarms 

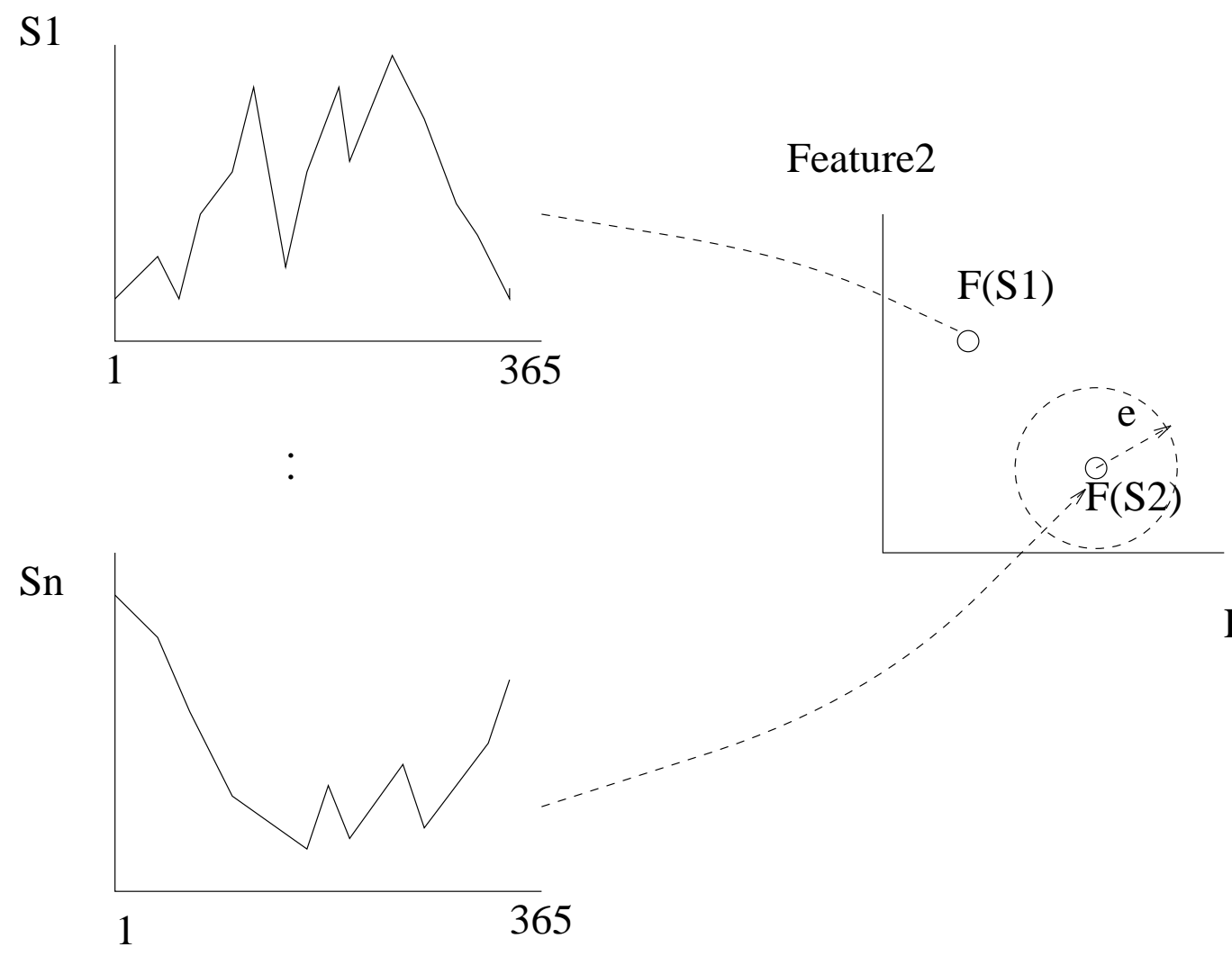

Feature1

Intuitively,

$$
D_{\text {feature }}\left(F\left(S_{1}\right), F\left(S_{2}\right)\right) \approx D_{\text {actual }}\left(S_{1}, S_{2}\right)
$$

Ideally, "=". However equality might be difficult (eg., 'dimensionality curse').

Lemma: To guarantee no false dismissals, lowerbound the actual distance:

$$
D_{\text {feature }}\left(F\left(S_{1}\right), F\left(S_{2}\right)\right) \leq D_{\text {actual }}\left(S_{1}, S_{2}\right)
$$

I.e., 'it is OK to make things look closer'. 
Solution for the whole-matching problem:

- perform Discrete Fourier Transform (DFT).

- keep first few coefficients

It works well, because:

- DFT maintains distances (Parseval's theorem)

- concentrates the 'energy', for 'colored noises'

- Keeping the first few coefficients lower-bounds the distance 
DFT formulas and definitions [Ham77] [OS75]:

$X_{f}=1 / \sqrt{n} \sum_{i=0}^{n-1} x_{i} \exp (-j 2 \pi f i / n) f=0,1, \ldots, n-1$

where $j=\sqrt{-1}$.

Energy of a signal $\vec{x}$ :

$$
E(\vec{x}) \equiv\|\vec{x}\|^{2} \equiv \sum_{i=0}^{n-1}\left|x_{i}\right|^{2}
$$

Theorem (Parseval).

$$
\sum_{i=0}^{n-1}\left|x_{i}\right|^{2}=\sum_{f=0}^{n-1}\left|X_{f}\right|^{2}
$$

and also:

$$
\|\vec{x}-\vec{y}\|^{2} \equiv\|\vec{X}-\vec{Y}\|^{2}
$$

The first few (eg., 2) coefficients result in a lower bound:

$\left(X_{1}-Y_{1}\right)^{2}+\left(X_{2}-Y_{2}\right)^{2} \leq\left(X_{1}-Y_{1}\right)^{2}+\left(X_{2}-Y_{2}\right)^{2}+\left(X_{3}-Y_{3}\right)^{2} \ldots$ 
Colored noises

- brown noise $\left(1 / f^{2}\right.$ energy spectrum $) \equiv$ random walk (stock price movements, currency exchange rates) [Mandelbrot] [Man77]

- pink noise (1/f energy spectrum) - works of art [Sch91]

- black noise $\left(1 / f^{b} b>2\right)$ water-level of rivers [Sch91] 


\section{Examples of colored noises:}
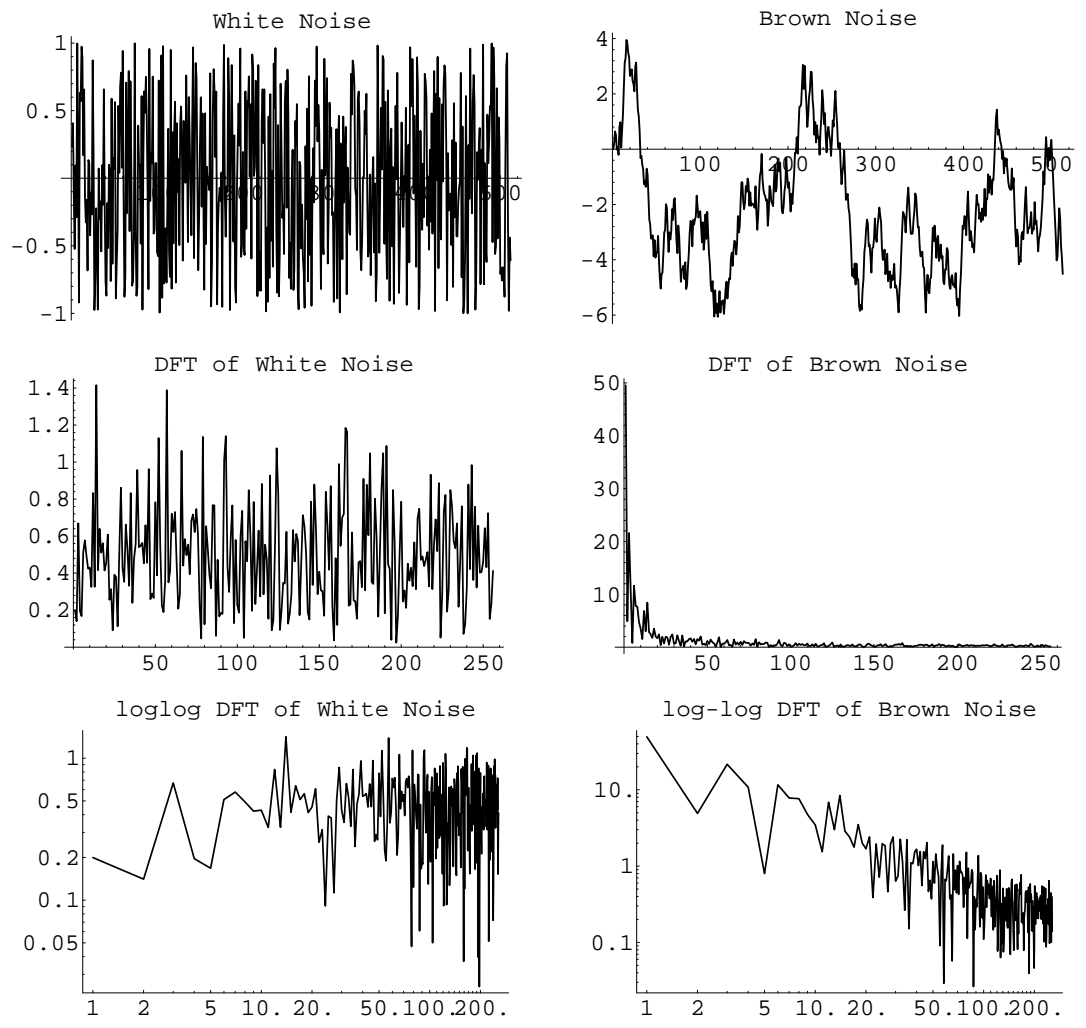

Other 'noises': skewed spectrum, too 


\section{Performance:}

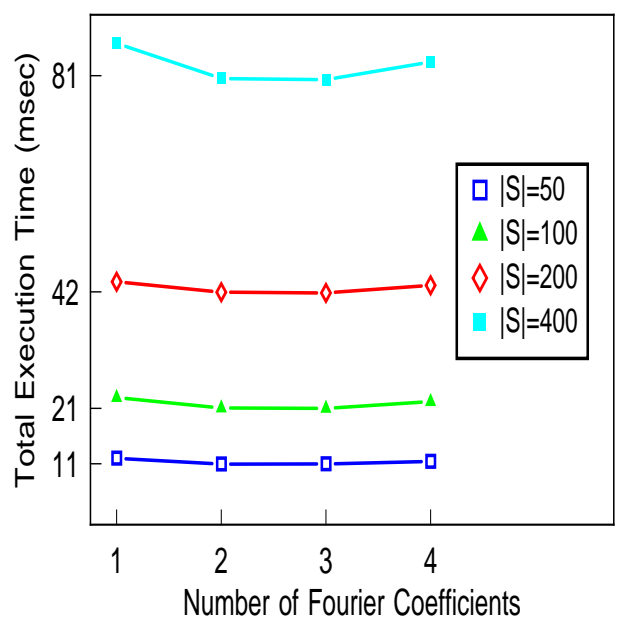

Figure 1: Time per query vs. \# Fourier coefficients, for range queries

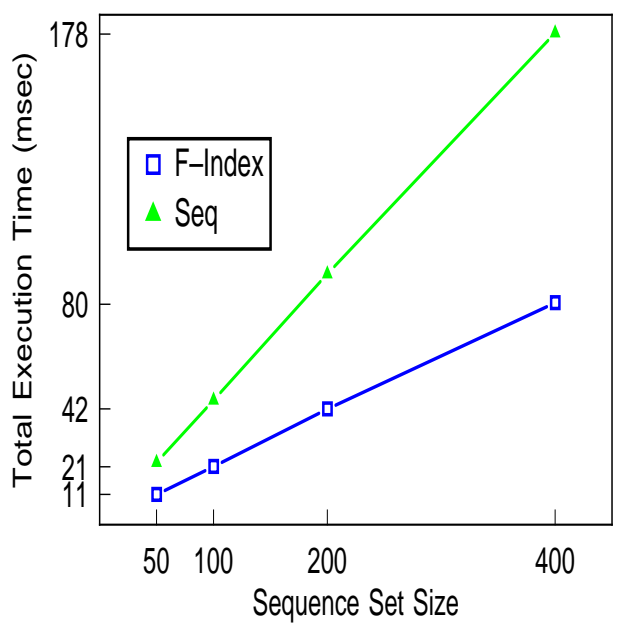

Figure 2: Time per query varying \# sequences, for range queries 


\section{COLOR IMAGES}

Much work on

- machine vision [BB82, DH73a], [TSSM89] [WSTM90] [CL91, CW92, LH90, LH92], [HK92], [IX90, Jag91a, $\mathrm{KKS}^{+}$91, CH91, MG89, GNM92, LW88], and [BGS92, SB91, Iok89];

- much work on fast searching;

- little communication between DB and MV communities [ACM91, JN92, $\mathrm{NBE}^{+}$93]

Except recently [HHLC92] [PO93] [?] $\left[\mathrm{FBF}^{+} 94\right]$ Goal: Queries on color, shape, texture, eg.,

- find photos with color distribution similar to a sunset over the ocean

- find shapes similar to tropical fish

Queries (a) 'by example' (b) 'by sketch' Support for combinations of color, shape, texture specifications; browsing; 'positional color'. 


\subsection{Color - features and distance func- tion}

COLOR IMAGE, eg. 256x256

i-th pixel:

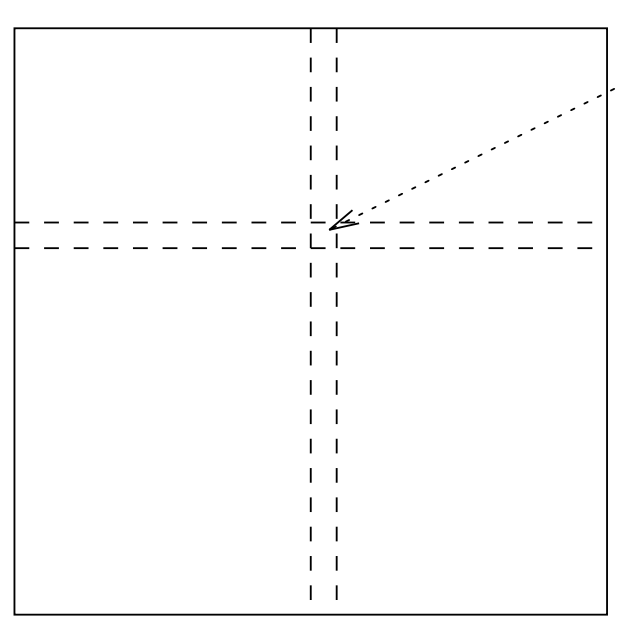

(ri, gi, bi)

$i$-th pixel: $\left(r_{i}, g_{i}, b_{i}\right)$ (Red, Green, Blue), $0 \leq r_{i}, g_{i}, b_{i} \leq$ 255

eg. $\operatorname{pink}=(200,60,60)( \pm)$

black $=(0,0,0)$

white $=(255,255,255)$ 
Feature vector: histogram with, say 64 colors (bright red, pink, orange, .... light blue, dark blue, ...)

Eg., sunset photo:

$$
\vec{x}=(80,85,75, \ldots, 90,110, \ldots)
$$
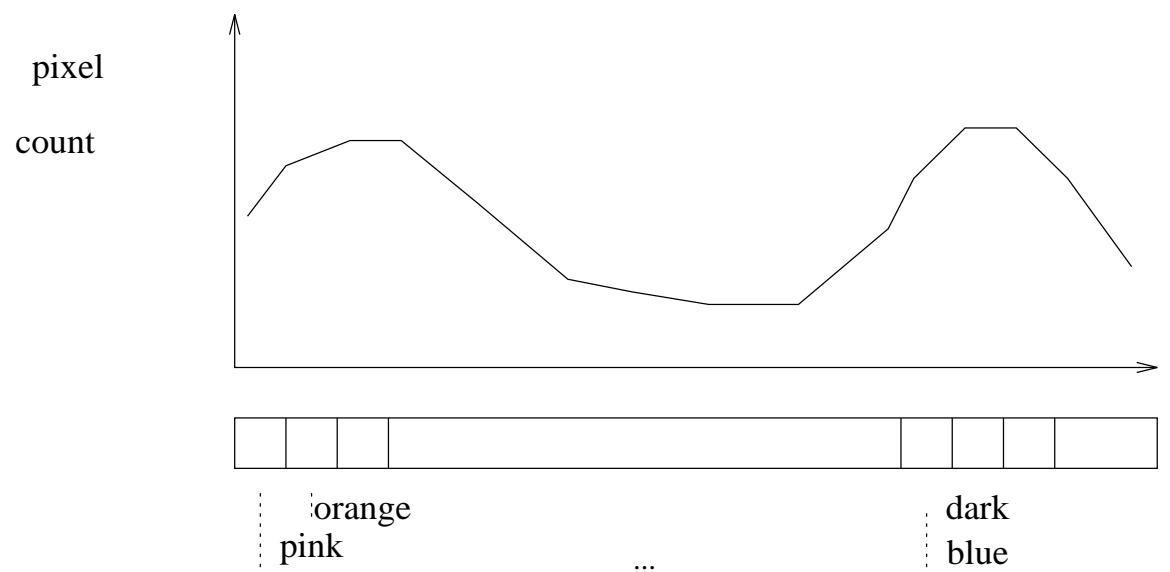

$\mathbf{x}$

bright

red

light

blue 
Distance of two histograms $\vec{x}$ and $\vec{q}$ : CROSS TALK bright red pink orange

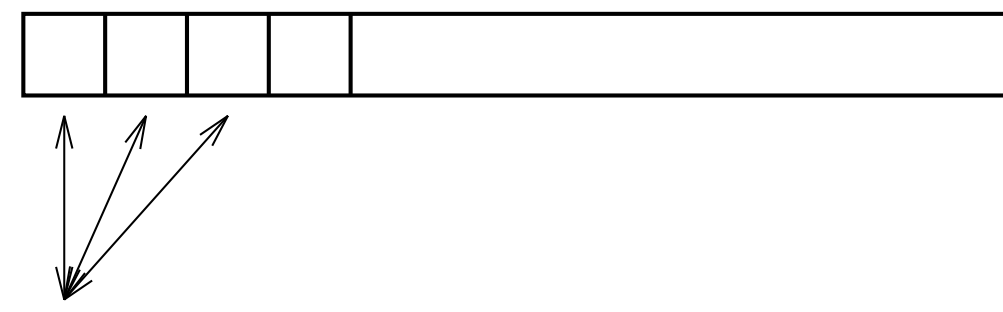
$\mathbf{x}$

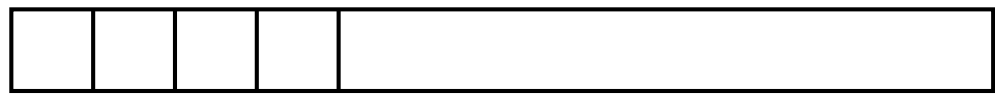

$\mathbf{q}$ eg, 64 colors

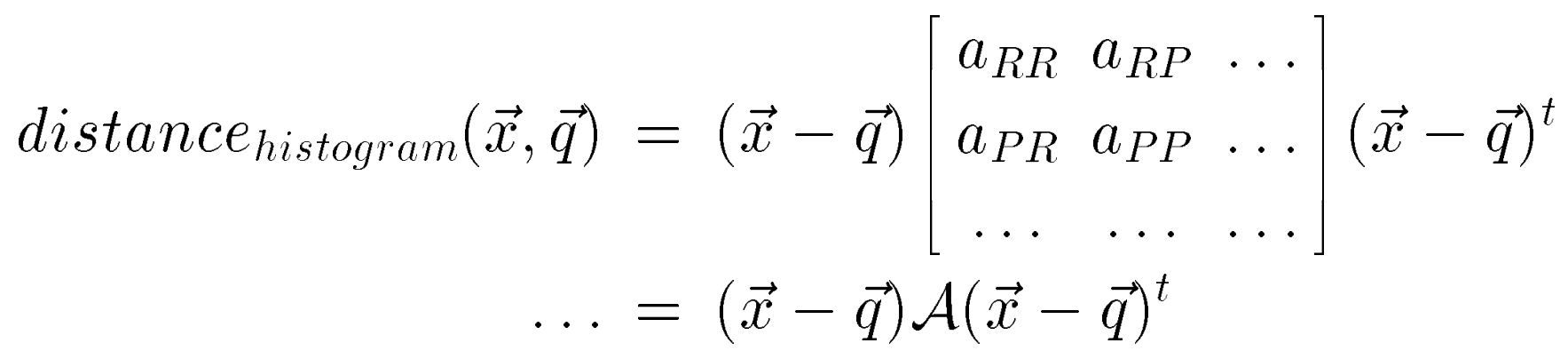


NONE of the S.A.Ms can handle crosstalk.

COLOR IMAGE, eg. 256x256

i-th pixel:

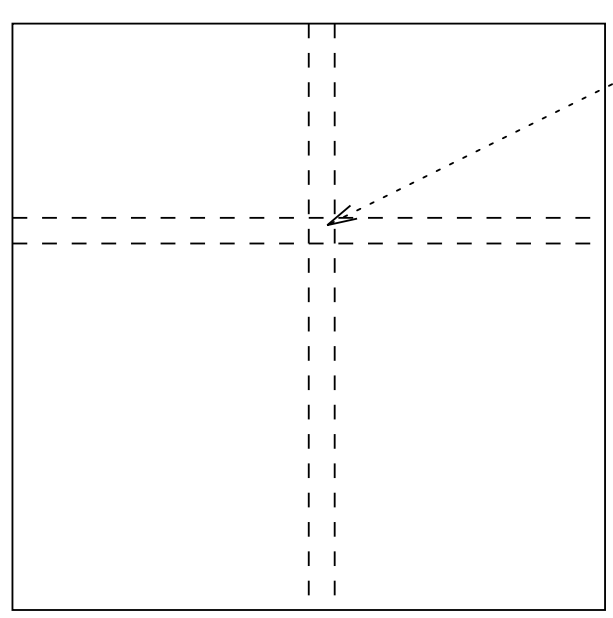

(ri, gi, bi)

Solution:

- Use a simpler feature vector, eg., average or total $\mathrm{R}, \mathrm{G}, \mathrm{B}$

$$
\overrightarrow{x^{\prime}}=\left(\sum r_{i}, \sum g_{i}, \sum b_{i}\right)
$$

with

$$
\text { distance }_{R G B}\left(\overrightarrow{x^{\prime}}, \overrightarrow{q^{\prime}}\right)=\text { Euclidean distance }
$$

- exploit the theorem $\left[\mathrm{FBF}^{+} 94\right]$

$$
\text { distance }_{R G B}\left(\overrightarrow{x^{\prime}}, \overrightarrow{q^{\prime}}\right) \leq \text { distance }_{\text {histogram }}(\vec{x}, \vec{q})
$$

Thus, the distance $_{R G B}()$ :

- is euclidean $\Rightarrow$ SAMs apply

- lowerbounds actual distance $\Rightarrow$ no false dismissals 


\section{$5.2 \quad$ Shapes}

- Features: area, perimeter, moments $(\approx 20)$

- Distance: (weighted) Euclidean

- Problem: too many features

- Solution: Karhunen-Loeve (K-L) transform ([Fuk90] $[\mathrm{DH} 73 \mathrm{~b}]) \Rightarrow$ 2-3 coefficients are enough

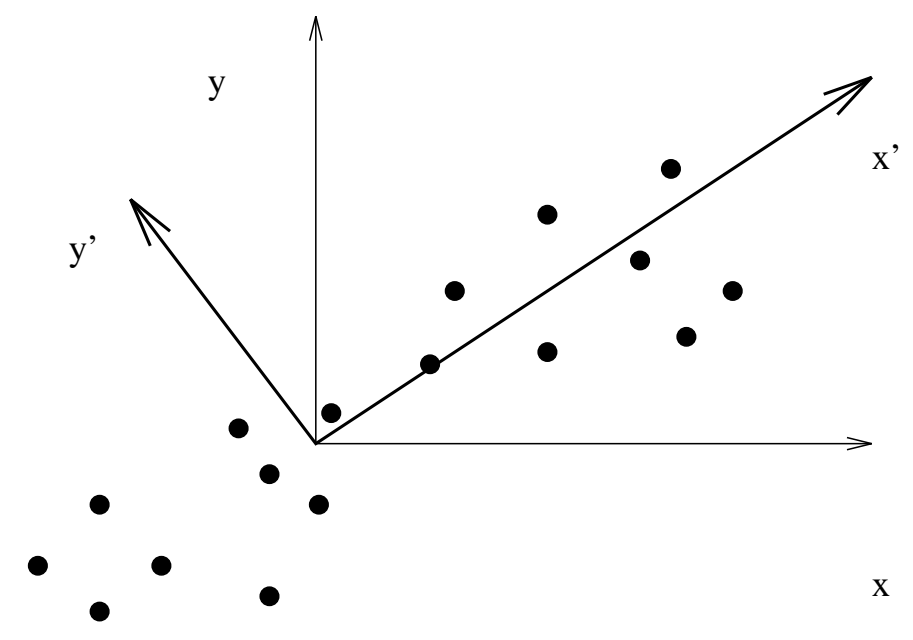




\subsection{Performance}

\section{For color}

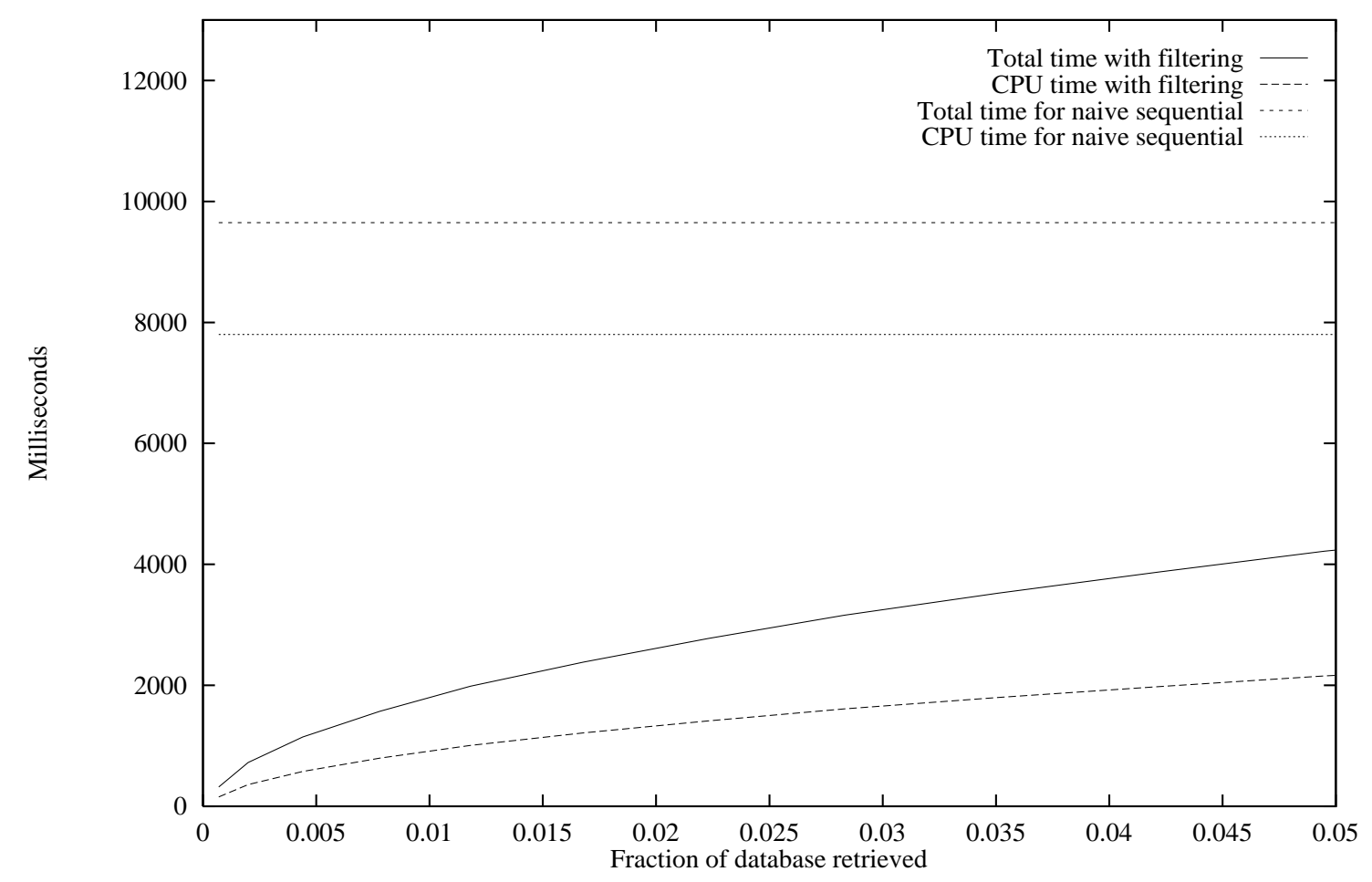

Figure 3: Time spent with sequential retrieval vs. filtered retrieval

\section{Observations: resolving crosstalk}

- allows indexing

- saves CPU time (distance histogram is $O\left(k^{2}\right)$ ). 


\section{Performance for shapes}

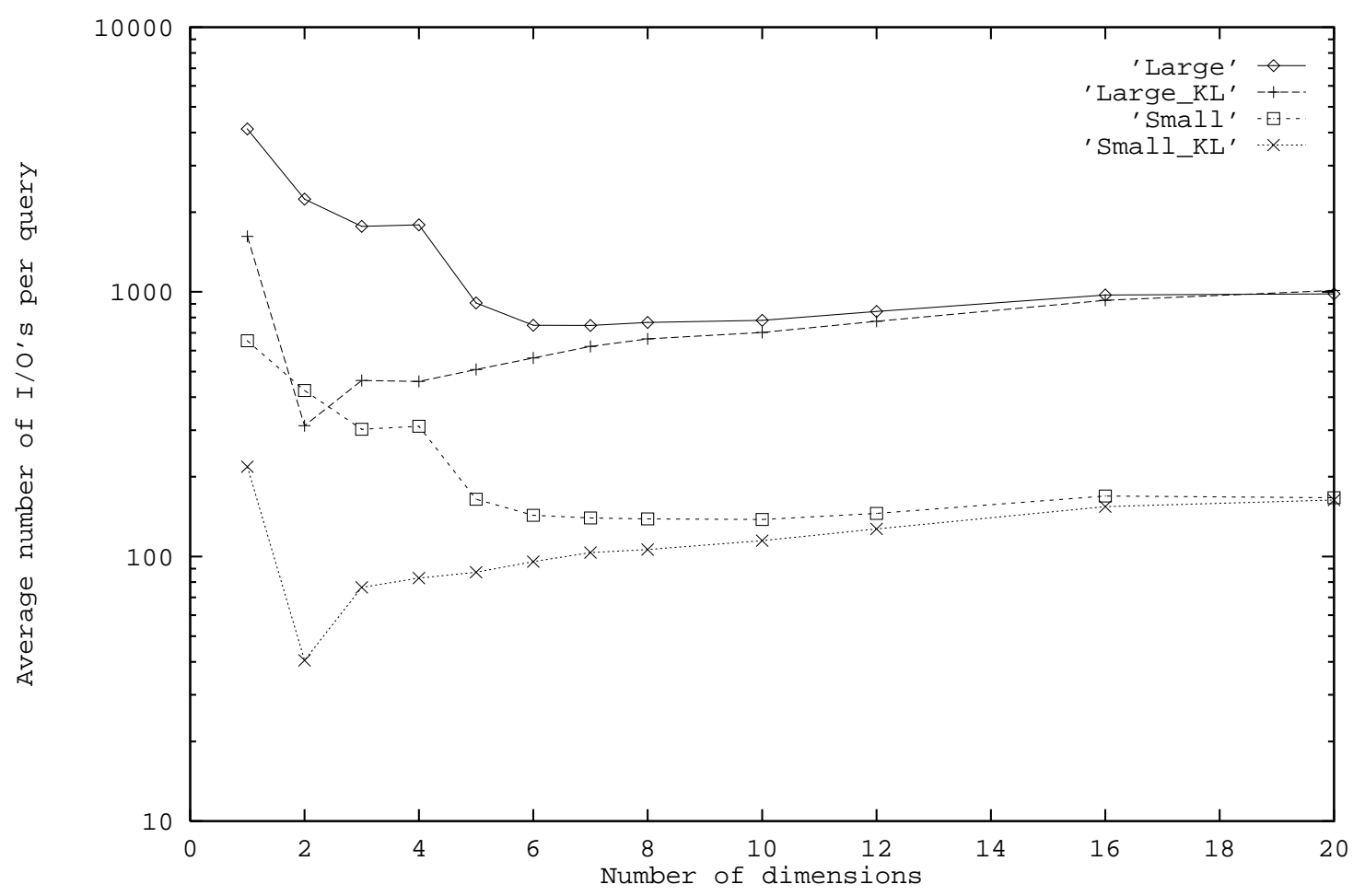

Figure 4: Average Disk I/O's per query, vs. dimensions kept

\section{Observations}

- first $2 \mathrm{~K}-\mathrm{L}$ coefficients are best ( $\approx 70 \%$ of energy)

- similar performance for larger db 


\section{3-D MEDICAL IMAGES}

Query by content in 2-d medical image databases [HHLC92] [PO93] [?]

Case study for 3-d medical images: QBISM [ $\left.\mathrm{ACF}^{+} 93\right]$ Goal: DB support for 3-d medical images (and specifically, for the Human Brain Mapping project). Ie.,:

given 3-d brain scans (PET, MRI etc) + demographic characteristics

build a system

to allow exploratory research

- a PET study: $(x, y, z$, intensity) tuples

- intensity $=$ glucose consumption rate $=$ brain activity

QBISM: Typical of multimedia retrieval system (handles scalar fields $=n$-d signals) 
Sample queries:

- fetch a patient's PET study

- show visual system

- rotate and/or slice

- show areas of high intensity

- list organs within $10 \mathrm{~mm}$ from hippocampus

- typical/average scan for 20-yr old left-handed females

- find brain scans similar to "Smith's" 


\section{System architecture:}

- DBMS, with 'long fields', extended SQL with contains, intersects etc., plus

- visualization package (eg., 'Data Explorer') as front end

DBMS

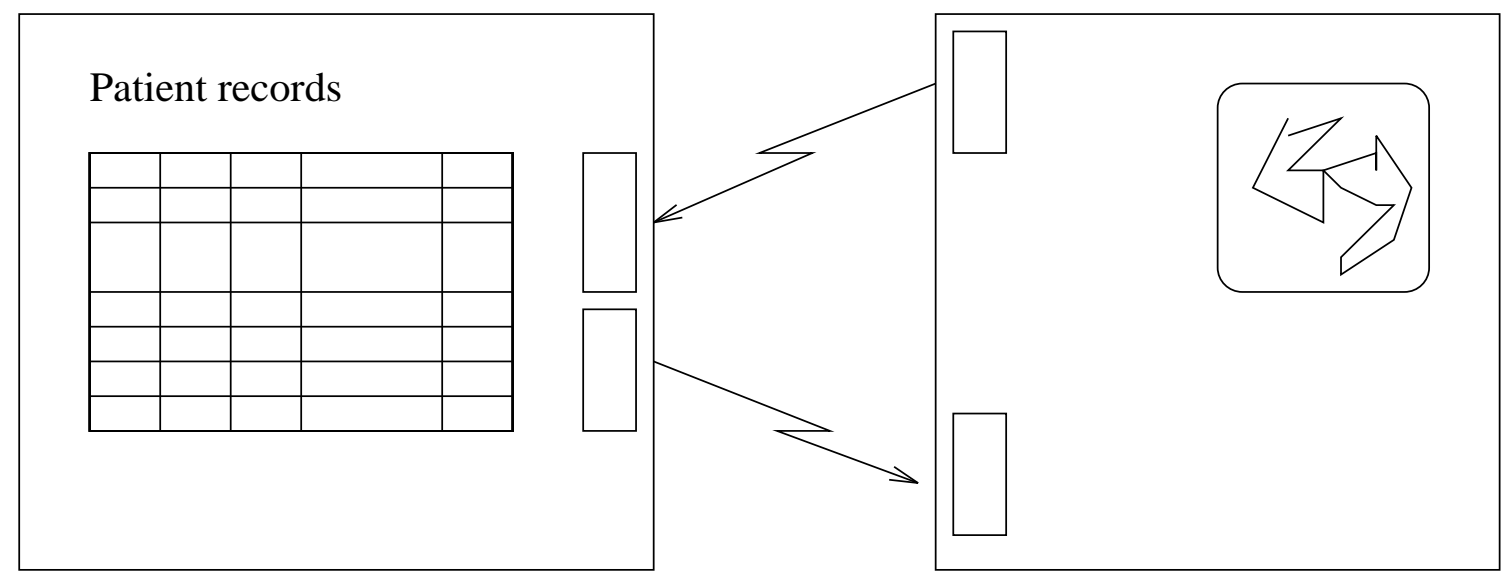


Good news for similarity searches:

- Euclidean distance is a good first step

- DFT leads to a skewed spectrum

Conclusions: Compared to a file-based system, a DBMS allows:

- convenient querying over multiple images

- better performance through early filtering 


\section{SUB-PATTERN MATCHING}

Problem: [Faloutsos et. al., SIGMOD 94] [FRM94]

- Given a collection of sequences (of variable duration)

- find the ones that contain a desirable pattern (within distance $\epsilon$ )

Database

S1

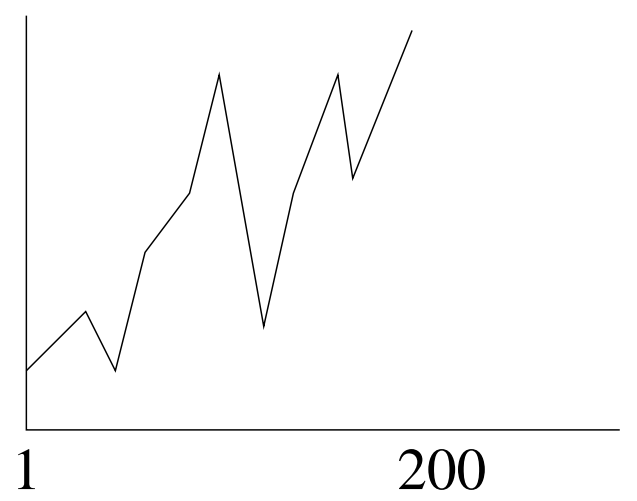

sub-sequence match

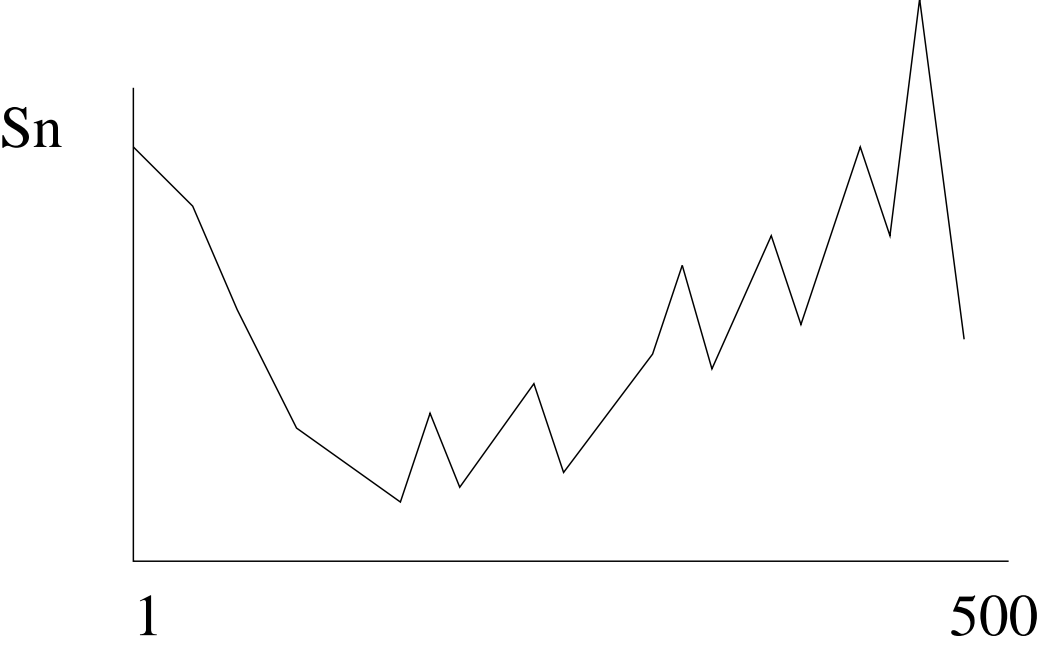


Assumption: queries have length $\geq w$

Proposed method: use sliding, overlapping windows to create trails in feature space.

Features: first few coefficients of the $w$-point DFT

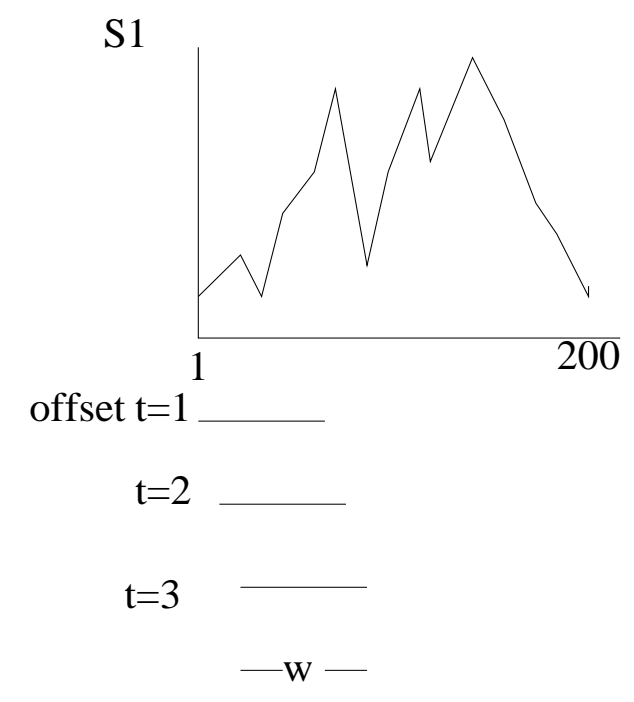

Feature2

(2nd DFT

coefficient)

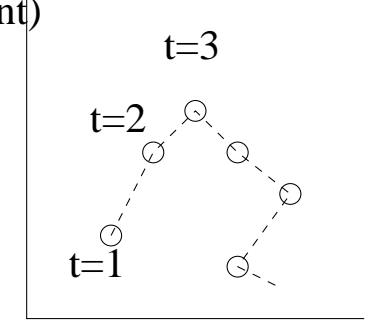

Feature1

(first coefficient

of \$w \$-point DFT)

$-\mathrm{W}$ 
More details on the method:

- subdivide trails and bound them by MBRs

- store MBRs in a S.A.M.

Q: Why not store all the points of the trail?

A: Too much space overhead!
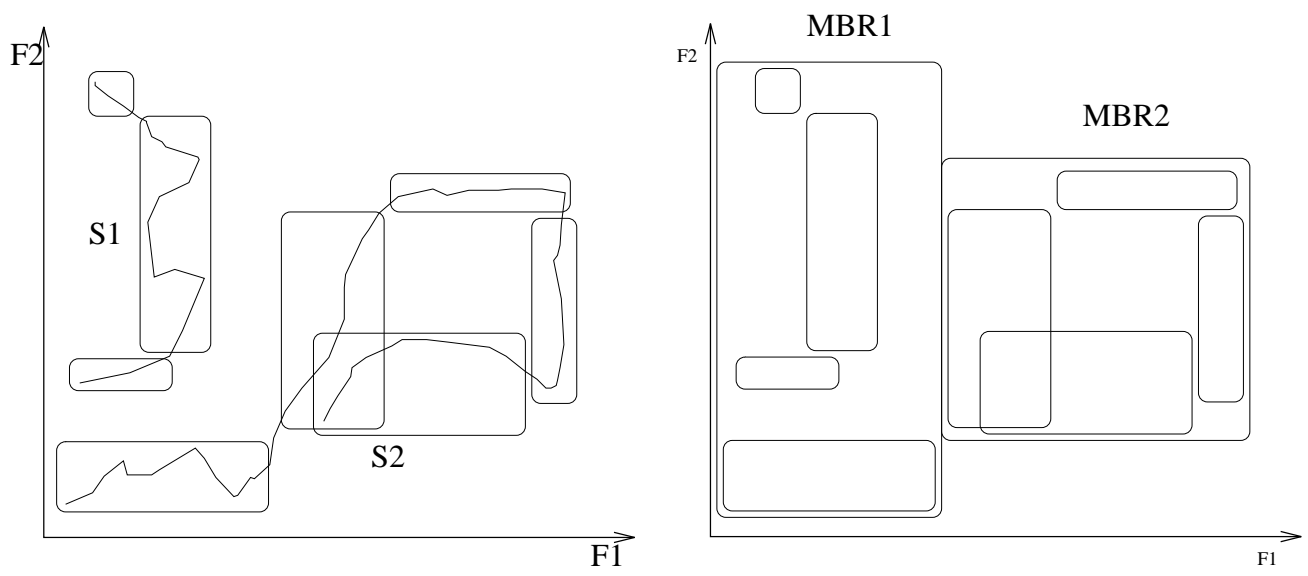

Leaf nodes: set of (seq-id, t-start, t-end, MBR)

Non-leaf nodes: set of (MBR, node-ptr) 
More details - how to divide a trail into subtrails?

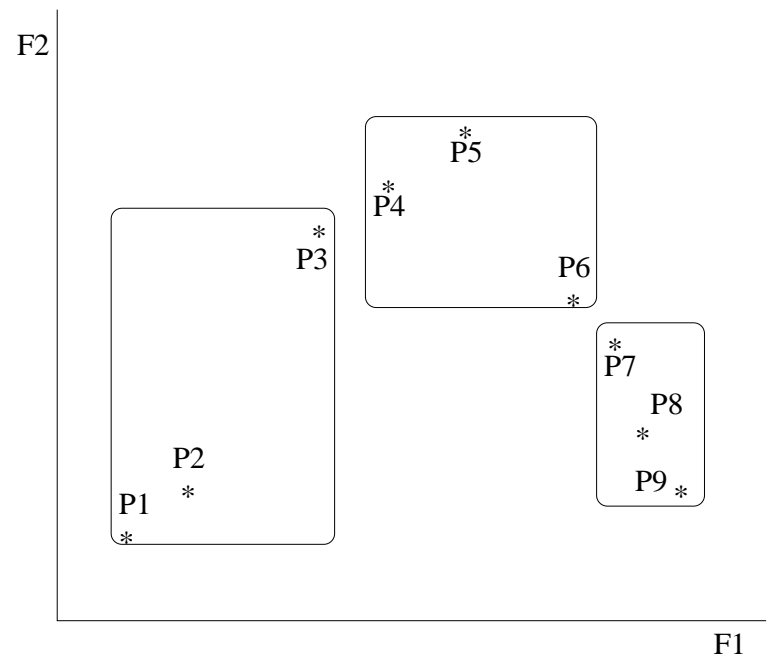

(a) 'fixed'

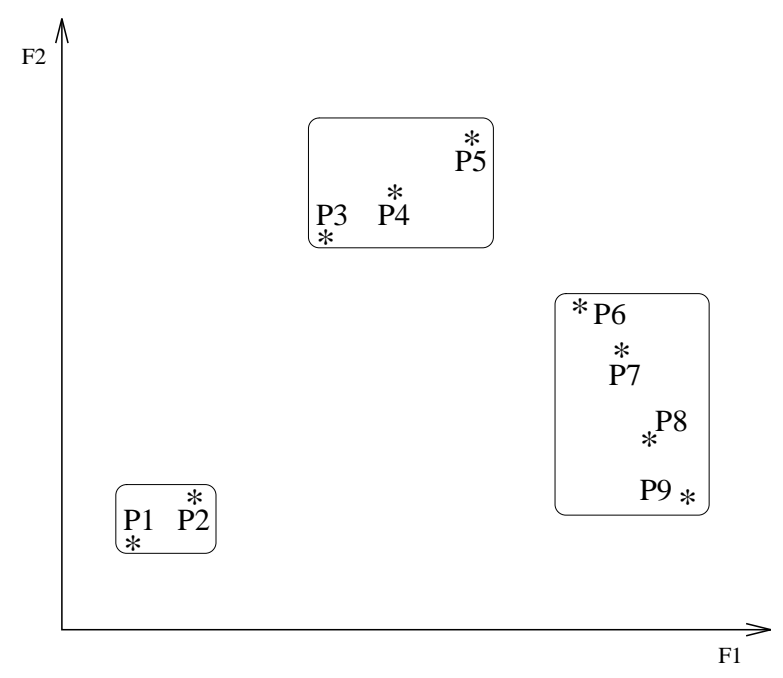

(b) 'adaptive' 


\section{Searching, for minimum-length queries:}

'short' query:
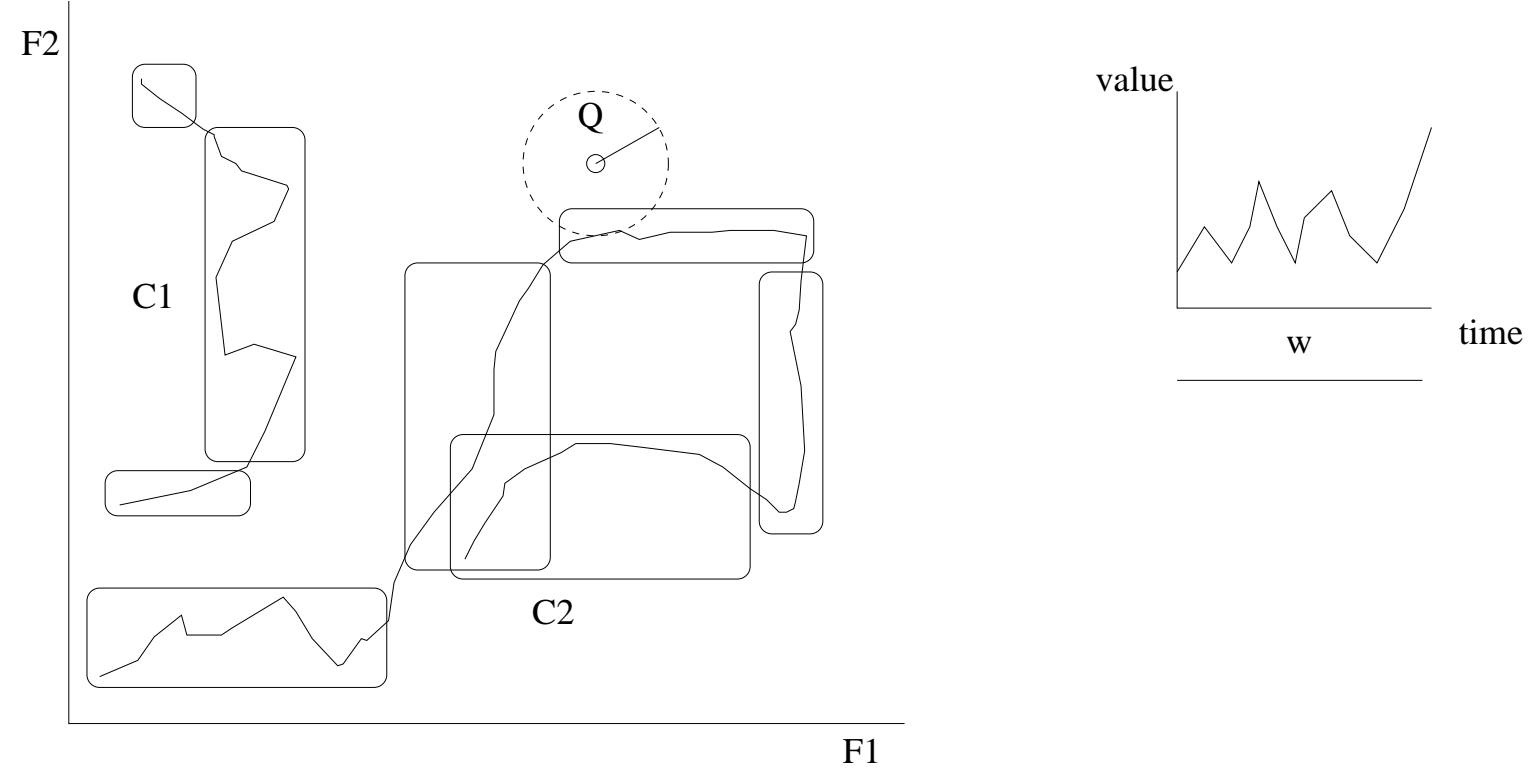

F1 
Searching, for longer queries: 'Multi-piece' algorithm:

- Break it in $p$ pieces of length $w$

- Search for each piece, with tolerance $\epsilon / \sqrt{p}$

- 'OR' the results and cleanup false alarms
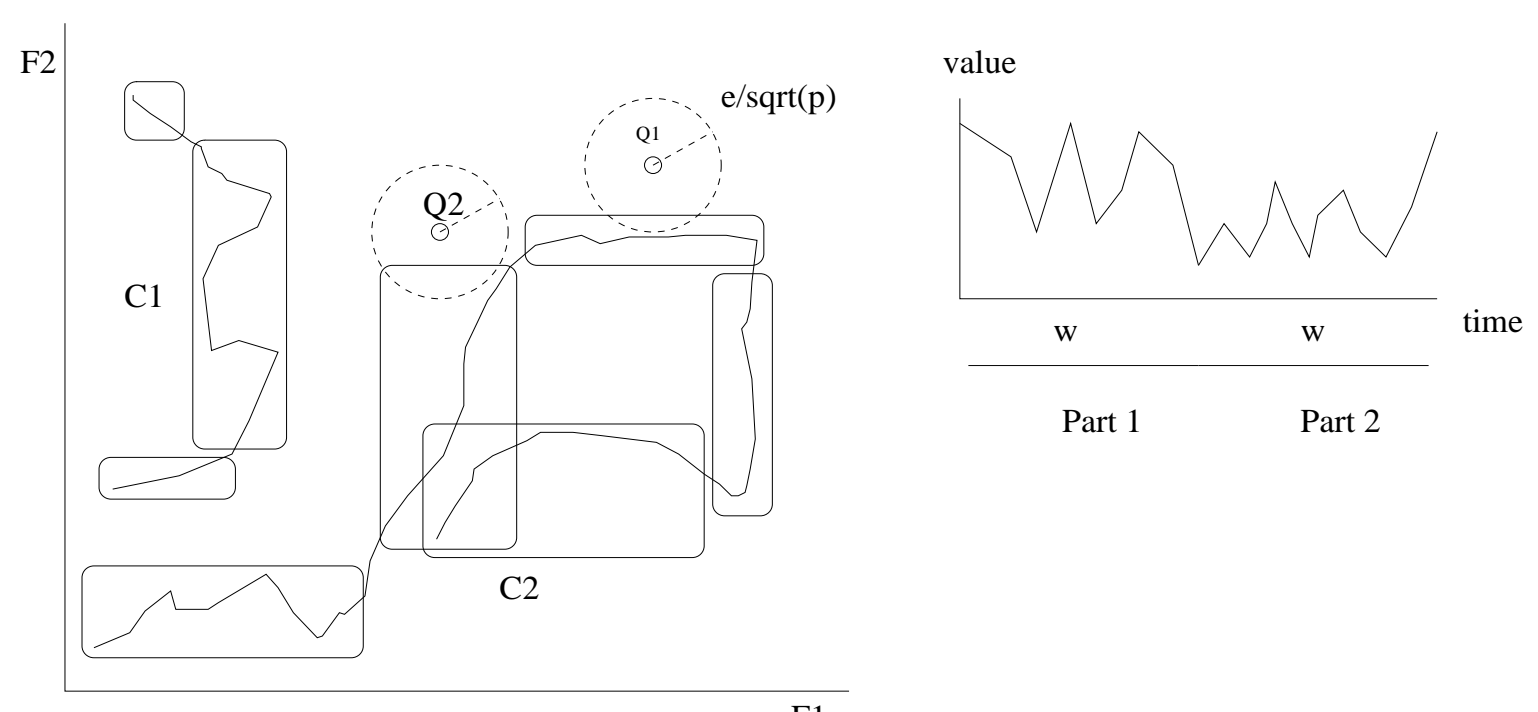

F1 


\section{Experiments}

- real data (stock prices) - 329,000 points; also, synthetic data.

- implementation in C, AIX, IBM RS/6000

- features: first 3 (complex) DFT coefficients

- 'adaptive' heuristic for sub-trails

- $\mathrm{R}^{*}$-tree [BKSS90] for S.A.M. 
Search time experiments - 'short' queries

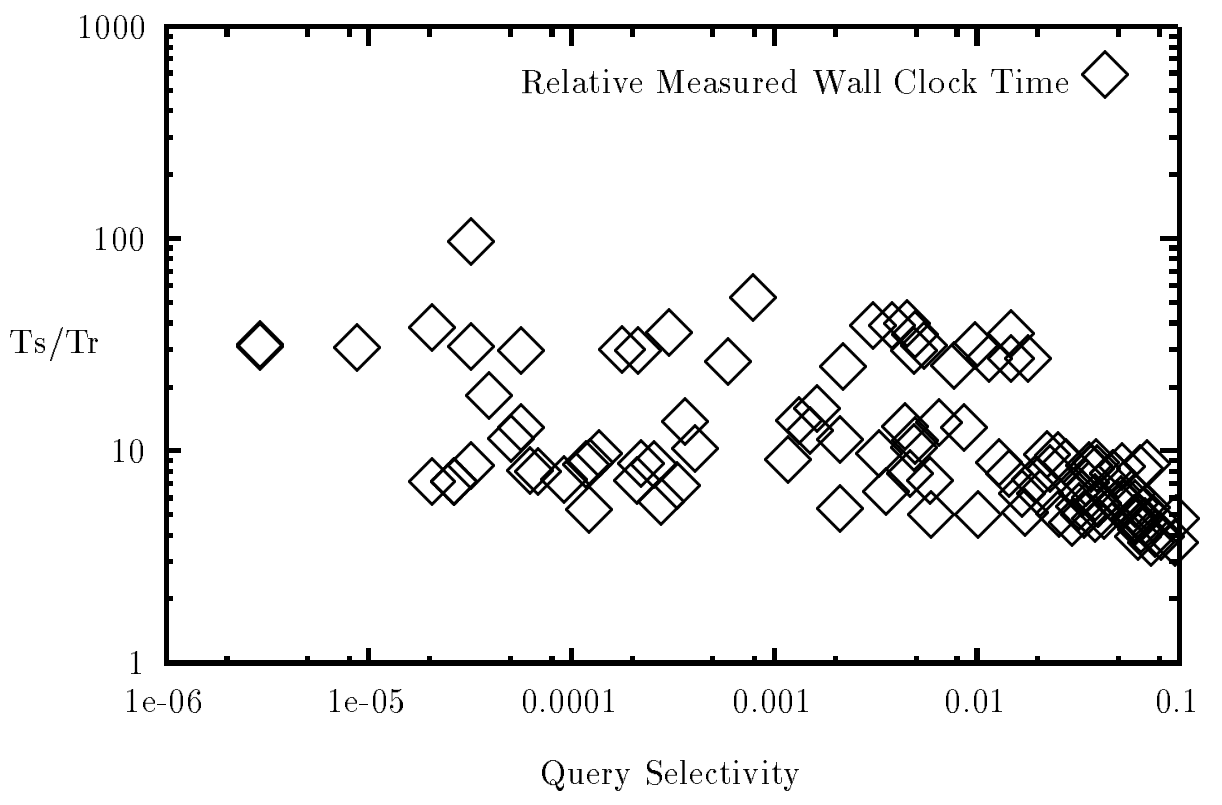

Relative wall clock time vs. selectivity in log-log scale $(\operatorname{Len}(Q)=w=512$ points $)$.

Conclusion: 3 to 100 times better than seq. scanning. 


\section{CONCLUSIONS}

Problem: Fast and 'complete' indexing for multimedia databases Solution: 'Quick and dirty' filter:

- map objects into points in feature space, lowerbounding the actual distance. $(\Rightarrow$ 'completeness').

- use any spatial access method ( $\Rightarrow$ efficiency).

Main idea, for 'sub-pattern matching'

- map an object into a set of (hyper)-rectangles in feature space

- use a S.A.M.

Occasional mismatches between features and SAMs:

- 'dimensionality curse': Use distance preserving, energy concentrating transforms (eg., DFT, DCT, wavelet $\left[\mathrm{RBC}^{+} 92\right]$ etc.)

- 'cross-talk': diagonalization 
Challenges:

- specific distance function / features for each application

- handling of non-Euclidean distance functions (eg., as in DNA strings) 


\section{References}

[ACF ${ }^{+93]}$ Manish Arya, William Cody, Christos Faloutsos, Joel Richardson, and Arthur Toga. Qbism: a prototype 3-d medical image database system. IEEE Data Engineering Bulletin, 16(1):38-42, March 1993.

[ACM91] ACM SIGIR. Proceedings of International Conference on Multimedia Information Systems, Singapore, 1991.

[AFS93] Rakesh Agrawal, Christos Faloutsos, and Arun Swami. Efficient similarity search in sequence databases. In Fourth Int. Conf. on Foundations of Data Organization and Algorithms (FODO), pages 69-84, Evanston, Illinois, October 1993. also available through anonymous ftp, from olympos.cs.umd.edu: ftp/pub/TechReports/fodo.ps.

[AIS93] Rakesh Agrawal, Tomasz Imielinski, and Arun Swami. Mining association rules between sets of items in large databases. Proc. ACM SIGMOD, pages 207-216, May 1993.

[BB82] D. Ballard and C. Brown. Computer Vision. Prentice Hall, 1982.

[BGS92] Elizabeth Binaghi, Isabella Gagliardi, and Raimondo Schettini. Indexing and fuzzy logic-based retrieval of color images. In Visual Database Systems, II, IFIP Transactions A- $\boldsymbol{7}$, pages 79-92. Elsevier Science Publishers, 1992.

[BKS93] Thomas Brinkhoff, Hans-Peter Kriegel, and Bernhard Seeger. Efficient processing of spatial joins using r-trees. Proc. of ACM SIGMOD, pages 237-246, May 1993.

[BKSS90] N. Beckmann, H.-P. Kriegel, R. Schneider, and B. Seeger. The r*-tree: an efficient and robust access method for points and rectangles. ACM SIGMOD, pages $322-$ 331, May 1990.

[CH91] Zen Chen and Shinn-Ying Ho. Computer vision for robust 3d aircraft recognition with fast library search. Pattern Recognition, 24(5):375-390, 1991.

[CL91] C. C. Chang and S. Y. Lee. Retrieval of similar pictures on pictorial databases. Pattern Recognition, 24(7):675-680, 1991.

[CW92] Chin-Chen Chang and Tzong-Chen Wu. Retrieving the most similar symbolic pictures from pictorial databases. Information Processing and Management, 28(5):581-588, 1992.

[DH73a] R. Duda and P Hart. Pattern Classification and Scene Analysis. Wiley, New York, 1973.

[DH73b] R.O. Duda and P.E. Hart. Pattern Classification and Scene Analysis. Wiley, New York, 1973. 
$\left[\mathrm{FBF}^{+}\right.$94] C. Faloutsos, R. Barber, M. Flickner, J. Hafner, W. Niblack, D. Petkovic, and W. Equitz. Efficient and effective querying by image content. Journal of Intell. Inf. Systems, 3(3/4):231-262, July 1994.

[FK94] Christos Faloutsos and Ibrahim Kamel. Beyond Uniformity and Irndependence: Analysis of R-trees Using the Concept of Fractal Dimension. Proc. ACM SIGACTSIGMOD-SIGART PODS, pages 4-13, May 1994. Also available as CS-TR-3198, UMIACS-TR-93-130.

[FR89a] C. Faloutsos and W. Rego. Tri-cell: a data structure for spatial objects. Information Systems, 14(2):131-139, 1989. early version available as UMIACS-TR-87-15, CS-TR-1829.

[FR89b] C. Faloutsos and S. Roseman. Fractals for secondary key retrieval. Eighth ACM SIGACT-SIGMOD-SIGART Symposium on Principles of Database Systems (PODS), pages 247-252, March 1989. also available as UMIACS-TR-89-47 and CS-TR-2242.

[FRM94] Christos Faloutsos, M. Ranganathan, and Yannis Manolopoulos. Fast subsequence matching in time-series databases. Proc. ACM SIGMOD, pages 419-429, May 1994. 'Best Paper' award; also available as CS-TR-3190, UMIACS-TR-93-131, ISR TR-93-86.

[FSR87] C. Faloutsos, T. Sellis, and N. Roussopoulos. Analysis of object oriented spatial access methods. Proc. ACM SIGMOD, pages 426-439 426-439, May 1987. also available as SRC-TR-87-30, UMIACS-TR-86-27, CS-TR-1781.

[Fuk90] Keinosuke Fukunaga. Introduction to Statistical Pattern Recognition. Academic Press, 1990. 2nd Edition.

[Gar82] I. Gargantini. An effective way to represent quadtrees. Comm. of ACM (CACM), 25(12):905-910, December 1982.

[GNM92] William I. Grosky, Peter Neo, and Rajiv Mehrotra. A pictorial index mechanism for model-based matching. Data and Knowledge Engineering, 8:309-327, 1992.

[Gun86] O. Gunther. The cell tree: an index for geometric data. Memorandum No. UCB/ERL M86/89, Univ. of California, Berkeley, December 1986.

[Gut84] A. Guttman. R-trees: a dynamic index structure for spatial searching. Proc. ACM SIGMOD, pages 47-57, June 1984.

[Ham77] Richard Wesley Hamming. Digital Filters. Prentice-Hall Signal Processing Series, Englewood Cliffs, N.J., 1977.

[HHLC92] Hou, Hsu, Liu, and Chiu. A content-based indexing technique using relative geometry features. SPIE 92, 1662:59-68, 1992. 
[HK92] Kyoji Hirata and Toshikazu Kato. Query by visual example. In Advances in Database Techonology EDBT '92, Third International Conference on Extending Database Technology, Vienna, Austria, March 1992. Springer-Verlag.

[HN83] K. Hinrichs and J. Nievergelt. The grid file: a data structure to support proximity queries on spatial objects. Proc. of the WG'83 (Intern. Workshop on Graph Theoretic Concepts in Computer Science), pages 100-113, 1983.

[HSW88] A. Hutflesz, H.-W. Six, and P. Widmayer. Twin grid files: Space optimizing access schemes. Proc. of ACM SIGMOD, pages 183-190, June 1988.

[Iok89] Mikihiro Ioka. A method of defining the similarity of images on the basis of color information. Technical report RT-0030, IBM Tokyo Research Lab, 1989.

[IX90] M. A. Ireton and C. S. Xydeas. Classification of shape for content retrieval of images in a multimedia database. In Sixth International Conference on Digital Processing of Signals in Communications, pages 111 - 116, Loughborough, UK, 2-6 Sept., 1990. IEE.

[Jag90] H.V. Jagadish. Linear clustering of objects with multiple attributes. ACM SIGMOD Conf., pages 332-342, May 1990.

[Jag91a] H. V. Jagadish. A retrieval technique for similar shapes. In International Conference on Management of Data, SIGMOD 91, pages 208-217, Denver, CO, May 1991. ACM.

[Jag91b] H.V. Jagadish. A retrieval technique for similar shapes. Proc. ACM SIGMOD Conf., pages 208-217, May 1991.

[JN92] Ramesh Jain and Wayne Niblack. NSF Workshop on Visual Information Management, February 1992.

[KF93] Ibrahim Kamel and Christos Faloutsos. On packing r-trees. Second Int. Conf. on Information and Knowledge Management (CIKM), November 1993.

[KF94] Ibrahim Kamel and Christos Faloutsos. Hilbert R-tree: An Improved R-tree Using Fractals. In Proceedings of VLDB Conference,, pages 500-509, Santiago, Chile, September 1994.

[KKS ${ }^{+91]}$ T. Kato, T. Kurita, H. Shimogaki, T. Mizutori, and K. Fujimura. A cognitive approach to visual interaction. In International Conference of Multimedia Information Systems, MIS'91, pages 109-120. ACM and National University of Singapore, January 1991.

[LH90] Suh-Yin Lee and Fang-Jung Hsu. 2d c-string: A new spatial knowledge representation for image database systems. Pattern Recognition, 23(10):1077-1087, 1990. 
[LH92] Suh-Yin Lee and Fang-Jung Hsu. Spatial reasoning and similarity retrieval of images using $2 \mathrm{~d}$ c-string knowledge representation. Pattern Recognition, 25(3):305$318,1992$.

[LW88] Yehezkel Lamdan and Haim J. Wolfson. Geometric hashing: A general and efficient model-based recognition scheme. In 2nd International Conference on Computer Vision (ICCV), pages 238-249, Tampa, Florida, 1988. IEEE.

[Man77] B. Mandelbrot. Fractal Geometry of Nature. W.H. Freeman, New York, 1977.

[MG89] Rajiv Mehrotra and William I. Grosky. Shape matching utilizing indexed hypotheses generation and testing. IEEE Transactions on Robotics and Automation, $5(1): 70-77,1989$.

[NBE $\left.{ }^{+} 93\right]$ W. Niblack, R. Barber, W. Equitz, M. Flickner, E. Glasman, D. Petkovic, P. Yanker, C. Faloutsos, and G. Taubin. The QBIC project: Querying images by content using color, texture, and shape. SPIE 1993 International Symposium on Electronic Imaging: Science 83 Technology, Conference 1908, Storage and Retrieval for Image and Video Databases, February 1993.

[NHS84] J. Nievergelt, H. Hinterberger, and K.C. Sevcik. The grid file: an adaptable, symmetric multikey file structure. ACM TODS, 9(1):38-71, March 1984.

[OM88] J.A. Orenstein and F.A. Manola. Probe spatial data modeling and query processing in an image database application. IEEE Trans. on Software Engineering, 14(5):611-629, May 1988.

[Ore86] J. Orenstein. Spatial query processing in an object-oriented database system. Proc. ACM SIGMOD, pages 326-336, May 1986.

[Ore89] J.A. Orenstein. Redundancy in spatial databases. Proc. of ACM SIGMOD Conf., May 1989.

[Ore90] J.A. Orenstein. A comparison of spatial query processing techniques for native and parameter spaces. Proc. of ACM SIGMOD Conf., pages 343-352, 1990.

[OS75] Alan Victor Oppenheim and Ronald W. Schafer. Digital Signal Processing. Prentice-Hall, Englewood Cliffs, N.J., 1975.

[PO93] Euripides G.M. Petrakis and Stelios C. Orphanoudakis. Methodology for the Representation, Indexing and Retrieval of Images by Content. Image and Vision Computing, 11(8):504-521, October 1993.

[PSTW93] B. Pagel, H. Six, H. Toben, and P. Widmayer. Towards an analysis of range query performance. Proc. of ACM SIGACT-SIGMOD-SIGART Symposium on Principles of Database Systems (PODS), pages 214-221, May 1993. 
[RBC+92] Mary Beth Ruskai, Gregory Beylkin, Ronald Coifman, Ingrid Daubechies, Stephane Mallat, Yves Meyer, and Louise Raphael. Wavelets and Their Applications. Jones and Bartlett Publishers, Boston, MA, 1992.

[RF91] Yi Rong and Christos Faloutsos. Analysis of the clustering property of peano curves. Techn. Report CS-TR-2792, UMIACS-TR-91-151, Univ. of Maryland, December 1991.

[RKV95] Nick Roussopoulos, Steve Kelley, and F. Vincent. Nearest Neighbor Queries. Proc. of ACM-SIGMOD, pages 71-79, May 1995.

[RL85] N. Roussopoulos and D. Leifker. Direct spatial search on pictorial databases using packed R-trees. Proc. ACM SIGMOD, May 1985.

[Sam90a] H. Samet. Applications of Spatial Data Structures Computer Graphics, Image Processing and GIS. Addison-Wesley, 1990.

[Sam90b] H. Samet. The Design and Analysis of Spatial Data Structures. Addison-Wesley, 1990.

[SB91] Michael J. Swain and Dana H. Ballard. Color indexing. International Journal of Computer Vision, 7(1):11-32, 1991.

[Sch91] Manfred Schroeder. Fractals, Chaos, Power Laws: Minutes From an Infinite Paradise. W.H. Freeman and Company, New York, 1991.

[SRF87] T. Sellis, N. Roussopoulos, and C. Faloutsos. The $r+$ tree: a dynamic index for multi-dimensional objects. In Proc. 13th International Conference on VLDB, pages 507-518, England, September 1987. also available as SRC-TR-87-32, UMIACSTR-87-3, CS-TR-1795.

[SSH86] M. Stonebraker, T. Sellis, and E. Hanson. Rule indexing implementations in database systems. In Proceedings of the First International Conference on Expert Database Systems, Charleston, SC, April 1986.

[SSN87] C.A. Shaffer, H. Samet, and R.C. Nelson. Quilt: a geographic information system based on quadtrees. Technical Report CS-TR-1885.1, Univ. of Maryland, Dept. of Computer Science, July 1987. to appear in the International Journal of Geographic Information Systems.

[TSSM89] Satoshi Tanaka, Mitsuhide Shima, Jun’ichi Shibayama, and Akira Maeda. Retrieval method for an image database based on topological structure. In Applications of Digital Image Processing, volume 1153, pages 318-327. SPIE, 1989.

[Vas93] Dimitris Vassiliadis. The input-state space approach to the prediction of auroral geomagnetic activity from solar wind variables. Int. Workshop on Applications of Artificial Intelligence in Solar Terrestrial Physics, September 1993. 
[Whi81] M. White. N-Trees: Large Ordered Indexes for Multi-Dimensional Space. Application Mathematics Research Staff, Statistical Research Division, U.S. Bureau of the Census, December 1981.

[WSTM90] Koji Wakimoto, Mitsuhide Shima, Satoshi Tanaka, and Akira Maeda. An intelligent user interface to an image database using a figure interpretation method. In 9th Int. Conference on Pattern Recognition, volume 2, pages 516-991, 1990. 